\title{
Respiratory symptoms in older people and their association with mortality
}

\author{
J Hewitt, L Smeeth, C J Bulpitt, A J Tulloch, A E Fletcher
}

Thorax 2005;60:331-334. doi: 10.1136/thx.2004.029579

\begin{abstract}
See end of article for authors' affiliations

.....................

Correspondence to: DrJ Hewitt, Centre for Ageing and Public Health, London School of Hygiene and Tropical Medicine, London WCIE 7HT, UK; Jonathan.Hewitt@|shtm. ac.uk
\end{abstract}

Received 3 June 2004 Accepted 26 December 2004

\begin{abstract}
Background: A study was undertaken to assess the prevalence of reported respiratory symptoms in a population aged over 75 years and their association with mortality.

Methods: A total of 14458 people aged 75 years and over participating in a trial of health screening of older people in general practice answered questions on three respiratory symptoms: cough, sputum production, and wheeze. The association of symptoms with mortality was examined for all cause and respiratory causes of death taking account of potential confounders.

Results: Coughing up phlegm in winter mornings had a prevalence of $27.0 \%$ (95\% confidence interval (CI) 26.8 to 27.2 ). Those with this symptom had an adjusted hazard ratio for all cause mortality of $1.35195 \%$ $\mathrm{Cl} 1.21$ to 1.50$), \mathrm{p}<0.001$ and for respiratory specific mortality of $2.01(95 \% \mathrm{Cl} 1.66$ to 2.41$), \mathrm{p}<0.001$. Phlegm at any time of the day in winter had a prevalence of $16.5 \%(95 \% \mathrm{Cl} 16.3$ to 16.7$)$ with hazard ratios for all cause and respiratory specific mortality of $1.28(95 \% \mathrm{Cl} 1.15$ to 1.42$)$ and $2.28(95 \% \mathrm{Cl} 1.92$ to 2.70$), \mathrm{p}<0.001$. Wheeze or whistling from the chest had a prevalence of $14.3 \%(95 \% \mathrm{Cl} 14.1$ to 14.5$)$ with hazard ratios of $1.45(95 \% \mathrm{Cl} 1.31$ to 1.61$)$ and 2.86 (95\% Cl 2.45 to 3.35), $\mathrm{p}<0.001$.

Conclusions: The prevalence of respiratory symptoms is widespread among elderly people and their presence is a strong predictor of mortality.
\end{abstract}

$\mathrm{R}$ espiratory disease and symptoms are common problems in older people. Respiratory disease affects both the quality of life in elderly individuals and morbidity, although estimates of the prevalence of respiratory symptoms vary. $^{2-5}$

Increased rates of mortality are associated with established respiratory disease ${ }^{6}$ but the effect of self-reported symptoms alone, as predictors of mortality, has not been assessed. Analyses were therefore undertaken to establish the prevalence of three respiratory symptoms in an elderly population and to examine their association with mortality.

\section{METHODS \\ Participants}

The MRC trial of assessment and management of elderly people in the community is a cluster randomised trial investigating approaches to multidimensional screening for people aged 75 and over. ${ }^{78}$ A representative sample of 106 family practices in Great Britain were selected, stratified by mortality experience (standardised mortality ratio) and Jarman index (an area deprivation measure indicator). Practices were randomised into "universal" and "targeted" screening. In the universal arm all patients aged over 75 (excluding those resident in nursing homes or with terminal illnesses) were invited to complete a detailed screening assessment. The health aspects of the detailed screening questionnaire were extensive, covering many areas. These included physical health, such as the Rose angina questionnaire, and mental health with a 30 point mini mental state examination. As well as questions, physical measurements were made including body mass index and other anthropometric data, pulse rate and blood pressure. The social status of individuals was assessed with various estimates of socioeconomic status and social isolation including the Carstairs index. Detailed drug histories and routine blood tests for haematology, urea and electrolytes were performed. Routine urine dipstick analysis was also performed. Of particular relevance to this study, respiratory symptoms, ${ }^{9}$ previous diagnoses of emphysema or asthma, and smoking history were recorded. In the "targeted" arm only selected patients completed a detailed assessment, so patients in the "universal" arm were only included in this analysis as a representative sample of community dwelling older people. Participants were recruited between 1994 and 1999. All gave written informed consent and ethical approval for the study was obtained from relevant ethics committees. The three respiratory questions were: ${ }^{9}$

- Do you usually bring up phlegm from your chest first thing in the morning in winter? (yes or no)

- Do you usually bring up phlegm from your chest during the day or night in winter? (yes or no)

- Does your chest sound wheezy or whistling on most days (or nights)? (yes or no)

Trial participants were followed up for mortality by the UK Office for National Statistics (ONS) who provided fact and cause of death using ICD9 coding. Mortality data collection is ongoing and currently complete until December 2003. In this study a maximum of 5 years follow up was assessed.

\section{Statistical methods}

The prevalence of positive responses to each of the questions was estimated overall by age, sex, smoking habit (life long non-smokers, ex-smokers and current smokers) and socioeconomic status as measured by the Carstairs index. The Carstairs index uses overcrowding, unemployment, low social class, and car ownership to calculate a deprivation measure by area. ${ }^{10}$ Participants were assigned to quintiles of Carstairs score, with 1 being the lowest quintile and 5 the highest. The association of each symptom with mortality was examined using Cox's proportional hazards regression. A forward fitting model was constructed incorporating potential confounders ${ }^{11}{ }^{12}$ into the model for all cause, respiratory specific, and cardiovascular specific mortality. The potential confounding and interaction terms tested were age, sex, Carstairs index, smoking history, alcohol intake, previous 
Table 1 Prevalence (with $95 \% \mathrm{Cl}$ ) of respiratory symptoms and their distribution in an elderly population

\begin{tabular}{|c|c|c|c|c|}
\hline & No & Phlegm in winter mornings (\%) & Phlegm at any time of day (\%) & Wheeze (\%) \\
\hline Total & 15122 & 27.0 (26.8 to 27.2$)$ & 16.5 (16.3 to 16.7$)$ & 14.3 (14.1 to 14.5$)$ \\
\hline Male & 5794 & $35.7(33.5$ to 37.9$)$ & 23.3 (21.2 to 25.5$)$ & $17.1(15.2$ to 19.1$)$ \\
\hline Female & 9328 & $21.6(20.0$ to 23.4$)$ & $12.2(10.8$ to 13.8$)$ & $12.6(11.3$ to 14.1$)$ \\
\hline \multicolumn{5}{|l|}{ Age (years) } \\
\hline $75-79$ & 7093 & 26.3 (24.3 to 28.3 ) & 16.1 (13.3 to 19.4$)$ & 14.4 (12.9 to 16.1$)$ \\
\hline $80-84$ & 4739 & $28.4(26.5$ to 30.4$)$ & $16.8(14.7$ to 19.1$)$ & 15.3 (13.9 to 16.7$)$ \\
\hline $85-89$ & 2432 & $26.8(24.3$ to 29.4$)$ & $16.9(15.1$ to 18.8$)$ & $13.3(11.3$ to 15.6$)$ \\
\hline $90+$ & 858 & $25.9(22.7$ to 29.5$)$ & $16.1(14.4$ to 18.0$)$ & $11.5(9.2$ to 14.3$)$ \\
\hline \multicolumn{5}{|l|}{ Smoking } \\
\hline Non-smokers & 6022 & $19.8(18.3$ to 21.4$)$ & $10.4(9.1$ to 11.8$)$ & 9.5 (8.9 to 9.2$)$ \\
\hline Ex-smokers & 7519 & 29.1 (27.0 to 31.3$)$ & 18.4 (16.6 to 20.3$)$ & $16.1(14.5$ to 17.9$)$ \\
\hline Current & 1506 & $44.8(41.1$ to 47.9$)$ & $30.6(27.1$ to 34.3$)$ & $23.8(21.1$ to 26.8$)$ \\
\hline Missing & 75 & & & \\
\hline \multicolumn{5}{|l|}{ Carstairs by quintile } \\
\hline lst & 3273 & 20.9 (18.5 to 23.5$)$ & $11.6(10.3$ to 13.1$)$ & $10.0(8.3$ to 12.1$)$ \\
\hline 2nd & 3691 & 25.2 (23.5 to 27.1$)$ & 15.7 (14.0 to 17.6$)$ & 12.5 (11.1 to 14.0$)$ \\
\hline $3 r d$ & 3161 & 27.0 (24.7 to 29.4$)$ & 16.1 (13.5 to 19.12$)$ & $14.0(12.2$ to 15.9$)$ \\
\hline 4 th & 2272 & $29.7(27.3$ to 32.4$)$ & 17.9 (15.4 to 20.7$)$ & 16.5 (14.3 to 19.0$)$ \\
\hline 5 th & 1469 & $31.9(28.8$ to 35.2$)$ & $18.4(14.1$ to 23.7$)$ & $18.3(16.1$ to 20.8$)$ \\
\hline Missing & 1256 & & & \\
\hline Diagnosed emphysema & 230 & 55.7 (50.3 to 61.0) & 39.1 (31.9 to 46.8 ) & 47.6 (40.7 to 54.6 ) \\
\hline Diagnosed asthma & 1411 & 45.3 (41.9 to 48.8 ) & $31.8(27.8$ to 36.1$)$ & 43.4 (38.9 to 48.0 ) \\
\hline
\end{tabular}

Table 2 Hazard ratios (with 95\% Cl) for respiratory symptoms in an elderly population

\begin{tabular}{|c|c|c|c|}
\hline & Phlegm in winter mornings & Phlegm at any time of day & Wheeze \\
\hline \multicolumn{4}{|l|}{ All cause mortality } \\
\hline Unadjusted HR & 1.45 (1.33 to 1.59$)$ & 1.44 (1.30 to 1.59$)$ & $1.59(1.45$ to 1.75$)$ \\
\hline Men & 1.38 (1.22 to 1.57$)$ & 1.38 (1.20 to 1.58$)$ & 1.63 (1.43 to 1.87$)$ \\
\hline Women & $1.60(1.35$ to 1.84$)$ & $1.36(1.20$ to 1.54$)$ & $1.48(1.30$ to 1.67$)$ \\
\hline Non-smokers & 1.24 (1.04 to 1.51$)$ & 1.18 (1.01 to 1.38$)$ & 1.46 (1.24 to 1.72$)$ \\
\hline Ex-smokers & $1.62(1.40$ to 1.87$)$ & 1.56 (1.36 to 1.79$)$ & 1.54 (1.32 to 1.80$)$ \\
\hline Current smokers & 1.44 (1.15 to 1.80$)$ & 1.25 (1.01 to 1.55$)$ & $1.68(1.36$ to 2.09$)$ \\
\hline Adjusted HR & 1.35 (1.21 to 1.50$)$ & 1.28 (1.15 to 1.42$)$ & $1.45(1.31$ to 1.61$)$ \\
\hline Men* & $1.22(1.08$ to 1.38$)$ & 1.26 (1.09 to 1.45$)$ & $1.53(1.33$ to 1.76$)$ \\
\hline Women* & 1.54 (1.34 to 1.79$)$ & 1.30 (1.15 to 1.48$)$ & $1.45(1.26$ to 1.65$)$ \\
\hline Non-smokers† & $1.19(0.98$ to 1.45$)$ & $1.10(0.94$ to 1.29$)$ & $1.42(1.19$ to 1.68$)$ \\
\hline Ex-smokers $†$ & $1.43(1.24$ to 1.66$)$ & $1.40(1.21$ to 1.61$)$ & $1.46(1.25$ to 1.72$)$ \\
\hline Current smokers† & $1.33(1.06$ to 1.66$)$ & $1.23(0.98$ to 1.54$)$ & 1.71 (1.33 to 2.20$)$ \\
\hline \multicolumn{4}{|c|}{ Respiratory cause mortality } \\
\hline Unadjusted HR & 2.31 (1.95 to 2.75$)$ & 2.73 (2.28 to 3.27$)$ & 3.06 (2.63 to 3.55$)$ \\
\hline Men & 2.41 (1.85 to 3.12$)$ & 2.45 (2.01 to 2.99$)$ & 3.42 (2.75 to 4.24$)$ \\
\hline Women & 2.03 (1.60 to 2.58$)$ & 2.77 (2.14 to 3.59$)$ & 2.71 (2.08 to 3.53$)$ \\
\hline Non-smokers & $1.24(0.86$ to 1.79$)$ & $1.62(1.10$ to 2.40$)$ & $1.97(1.28$ to 3.03$)$ \\
\hline Ex-smokers & $2.87(2.25$ to 3.64$)$ & $2.96(2.35$ to 3.75$)$ & $3.10(2.52$ to 3.80$)$ \\
\hline Current smokers & $1.81(1.12$ to 2.92$)$ & $2.39(1.58$ to 3.60$)$ & $3.67(2.53$ to 5.35$)$ \\
\hline Adjusted HR & 2.01 (1.66 to 2.41$)$ & 2.28 (1.92 to 2.70$)$ & 2.86 (2.45 to 3.35$)$ \\
\hline Men $\ddagger$ & $2.11(1.57$ to 2.85$)$ & $1.97(1.60$ to 2.42$)$ & $2.94(2.31$ to 3.77$)$ \\
\hline Women $\ddagger$ & $1.87(1.46$ to 2.40$)$ & $2.74(2.10$ to 3.59$)$ & 2.71 (2.08 to 3.53$)$ \\
\hline Non-smokers§ & $1.22(0.84$ to 1.77$)$ & 1.79 (1.15 to 2.78$)$ & 2.15 (1.34 to 3.44$)$ \\
\hline Ex-smokers§ & 2.51 (1.95 to 3.24$)$ & 2.54 (2.02 to 3.22$)$ & 2.94 (2.35 to 3.67$)$ \\
\hline Current smokers§ & 1.74 (1.02 to 2.97$)$ & $2.13(1.37$ to 3.30$)$ & $3.49(2.27$ to 5.36$)$ \\
\hline \multicolumn{4}{|c|}{ Cardiovascular cause mortality } \\
\hline Unadjusted HR & $1.36(1.19$ to 1.55$)$ & 1.42 (1.25 to 1.62$)$ & 1.45 (1.24 to 1.70$)$ \\
\hline Men & 1.20 (1.02 to 1.39$)$ & $1.18(0.99$ to 1.40$)$ & $1.45(1.14$ to 1.84$)$ \\
\hline Women & $1.44(1.23$ to 1.68$)$ & $1.60(1.32$ to 1.94$)$ & 1.38 (1.14 to 1.67$)$ \\
\hline Non-smokers & $1.18(0.97$ to 1.43$)$ & $1.25(0.99$ to 1.59$)$ & $1.28(0.99$ to 1.64$)$ \\
\hline Ex-smokers & $1.44(1.21$ to 1.71$)$ & 1.50 (1.24 to 1.82$)$ & $1.50(1.22$ to 1.85$)$ \\
\hline Current smokers & $1.37(0.99$ to 1.90$)$ & $1.27(0.91$ to 1.76$)$ & $1.38(0.97$ to 1.96$)$ \\
\hline Adjusted HR & 1.26 (1.12 to 1.42$)$ & 1.31 (1.15 to 1.49$)$ & 1.37 (1.17 to 1.60$)$ \\
\hline Men* & $1.12(0.95$ to 1.32$)$ & $1.10(0.93$ to 1.31$)$ & 1.38 (1.09 to 1.76$)$ \\
\hline Women* & $1.42(1.21$ to 1.68$)$ & $1.64(1.34$ to 2.00$)$ & 1.35 (1.11 to 1.67$)$ \\
\hline Non-smokers $†$ & $1.12(0.90$ to 1.38$)$ & $1.25(0.98$ to 1.60$)$ & $1.23(0.95$ to 1.61$)$ \\
\hline Ex-smokers $†$ & $1.33(1.10$ to 1.60$)$ & $1.40(1.14$ to 1.71$)$ & $1.41(1.15$ to 1.73$)$ \\
\hline Current smokerst & $1.35(0.95$ to 1.93$)$ & $1.11(0.80$ to 1.53$)$ & $1.43(0.97$ to 2.10$)$ \\
\hline
\end{tabular}

*Adjusted for age group, smoking, Carstairs index, and previous myocardial infarction. Stratified by sex. †Adjusted for sex, age group, Carstairs index, and previous myocardial infarction. Stratified by smoking. $\ddagger$ Adjusted for age group, smoking, and Carstairs index. Stratified by sex.

$\S$ Adjusted for sex, age group, and Carstairs index. Stratified by smoking. 
myocardial infarction, and previous cerebrovascular accident. The questionnaire did not contain information regarding specific respiratory confounders such as family history of airways disease or detailed occupational history. Each model was then tested to ensure the proportional hazards assumption was maintained. All analyses were undertaken using STATA statistical software Version 7, taking into account the clustered study design of practice selection.

\section{RESULTS}

In total, 15122 of 21410 eligible patients (70.6\%) participated in the "universal" arm of the trial and underwent the detailed assessment; 14458 individuals answered the questions about respiratory symptoms. Overall, $27.0 \%$ reported phlegm in the winter mornings, $16.5 \%$ phlegm at anytime in winter, and $14.3 \%$ wheeze (table 1). Each symptom was strongly associated with a previous diagnosis of emphysema or asthma $(\mathrm{p}<0.001)$. The prevalence of each symptom was higher in men and among ex and current smokers, and increased with decreasing quintile of the Carstairs index $(\mathrm{p}<0.001)$. A total of 3217 people died $(565$ of respiratory causes) over a mean follow up period of 4.1 years (range 3.8 months to 5 years). There was a total of 63287 personyears of follow up. The overall unadjusted death rate was 47.75 per 1000 per year (95\% CI 44.83 to 50.89 ).

The reporting of any respiratory symptom increased the hazard ratios for all cause mortality and respiratory mortality with 2-3-fold risks for adjusted respiratory mortality (all $\mathrm{p}<0.001)$. The adjusted respiratory mortality hazard ratios were consistently higher than both all cause and cardiovascular mortality hazard ratios, which were similar (table 2 ). Adjusted mortality rates stratified by sex did not differ for any symptom. Stratification for smoking led to different results. Ex-smokers and current smokers had higher rates for phlegm in the winter morning and phlegm at any time of the day than lifelong non-smokers, but the hazard ratios for both all cause and respiratory mortality were similar to each other. The hazard ratios for wheeze increased continuously from non-smokers to ex-smokers to current smokers.

\section{DISCUSSION}

The prevalence estimates reported in this study constitute the largest estimate of respiratory symptoms among older people and the first time these symptoms have been used as predictors of mortality in an elderly population.

The prevalence of phlegm in winter mornings has previously been found to be $27.2 \%$ in men and $14.6 \%$ in women in a group aged $60-70$ years. ${ }^{6}$ The results obtained from our study (35.7\% for men and $21.6 \%$ for women) suggest that phlegm in winter mornings is more prevalent in later life. Previous survey estimates of the prevalence of daily sputum production are around 36\% in individuals aged over 45 years $^{5}$ and $22 \%$ in a slightly older group of people (aged up to 66 years)..$^{13}$ In our study the prevalence of phlegm at any time of the day or night during winter was $16.5 \%$ (95\% CI 16.3 to 16.7 ), less than in younger age groups; $14.5 \%$ is the first estimate for daily wheeze as a symptom in this age group.

Sputum production and wheeze are likely to reflect underlying chronic obstructive airways disease. The strong associations found between respiratory symptoms and previous diagnoses of asthma and emphysema support this. In addition, asthma is recognised as being underdiagnosed in this age group ${ }^{14}$ and this will be the underlying diagnosis in some individuals with wheeze not previously diagnosed with asthma.

The mortality rates for both all cause mortality and respiratory mortality when stratified by smoking groups for phlegm in the winter mornings and phlegm at any time of the day are interesting. Both show ex-smokers and current smokers to have a similar hazard ratio. The reasons for this are unclear. It is possible that this symptom is a good marker for underlying disease in ex-smokers or that there is less benefit in smoking cessation in individuals with either of these two symptoms. In addition, many smokers will have died before the age of 75 years and it is possible that elderly smokers may be less susceptible to certain symptoms caused by smoking and smoking related disease. The hazard ratio for wheeze increased with each smoking group, suggesting a continued negative effect of smoking.

The increased hazard ratios show that, in older people, respiratory symptoms are very strong predictors of death. The mortality hazard ratios were higher for respiratory death than for cardiovascular death. This implies that respiratory symptoms are particularly good indicators of respiratory mortality, although the lack of formal lung function testing in this study to support this must be noted as a limitation. However, it is unlikely that reporting bias is an explanatory factor because of the high predictive association with respiratory death. Another limitation is the response rate of the questionnaire. The response rate of over $70 \%$ is very good. Nonetheless, it is well established from epidemiological surveys that the $30 \%$ of non-responders are more likely to have poor health and less health seeking behaviour.

As respiratory symptoms predict mortality, it would seem sensible to recommend treatment on the basis of symptoms alone. In the elderly population, where respiratory function testing can be difficult and unreliable, this would seem especially so. Smoking cessation advice and treatment should be offered to all who continue to smoke, particularly in those with a wheeze.

Our results form the largest and most representative study of respiratory symptoms undertaken in people aged 75 years and above. They show that the prevalence of respiratory symptoms is widespread. These respiratory symptoms are not benign. People with any of these symptoms have increased rates of mortality and the raised hazard ratios can be used as predictors of respiratory death.

\section{ACKNOWLEDGEMENT}

The authors thank Chris Grundy for providing the Carstairs data.

\section{Authors' affiliations}

J Hewitt, L Smeeth, A E Fletcher, Centre for Ageing and Public Health, London School of Hygiene and Tropical Medicine, London, UK C J Bulpitt, Section of Care of the Elderly, Imperial College Faculty of Medicine, Hammersmith Campus, London, UK

A J Tulloch, Unit of Health Care Epidemiology, Institute of Health Sciences, University of Oxford, Oxford, UK

Funding was provided for the main trial by the Medical Research Council, the Department of Health, and the Scottish Office. Jonathan Hewitt is funded by NHS Research and Development, Eastern Region. Liam Smeeth was supported by the Medical Research Council.

Conflict of interest: none.

\section{REFERENCES}

1 Renwick DS, Connolly MJ. Impact of obstructive airways disease on quality of life in older adults. Thorax 1996;51:520-5.

2 Dow L, Fowler L, Phelps L, et al. Prevalence of untreated asthma in a population sample of 6000 older adults in Bristol, UK. Thorax 2001;56:472-6.

3 Horsley JR, Sterling IJ, Waters WE, et al. Respiratory symptoms among elderly people in the New Forest area as assessed by postal questionnaire. Age Ageing 1991;20:325-31.

4 Lundback B, Stjernberg N, Nystrom L, et al. An interview study to estimate prevalence of asthma and chronic bronchitis. The obstructive lung disease in northern Sweden study. Eur J Epidemiol 1993;9:123-33.

5 Renwick DS, Connolly MJ. Prevalence and treatment of chronic airways obstruction in adults over the age of 45. Thorax 1996;51:164-8.

6 Littlejohns P, Ebrahim S, Anderson R. Prevalence and diagnosis of chronic respiratory symptoms in adults. BMJ 1989;298:1556-60. 
7 Fletcher $\mathrm{AE}$, Jones DA, Bulpitt $\mathrm{CJ}$, et al. The MRC trial of assessment and management of older people in the community: objectives, design and interventions (ISRCTN23494848). BMC Health Serv Res 2002;2:21.

8 Smeeth L, Fletcher AE, Stirling S, et al. Randomised comparison of three methods of administering a screening questionnaire to elderly people: findings from the MRC trial of the assessment and management of older people in the community. BMJ 2001;323:1403-7.

9 Fletcher CM. Standardised questions on respiratory symptoms. Lancet 1960, 1665.

10 Carstairs V, Morris R. Deprivation: explaining differences in mortality between Scotland and England and Wales. BMJ 1989;299:886-9.
11 Sobradillo V, Miravitlles M, Jimenez CA, et al. [Epidemiological study of chronic obstructive pulmonary disease in Spain (IBERPOC): prevalence of chronic respiratory symptoms and airflow limitation]. Arch Bronconeumol 1999:35:159-66.

12 Mannino DM. COPD: epidemiology, prevalence, morbidity and mortality, and disease heterogeneity. Chest 2002;121:121-6S.

13 Lundback B, Nystrom L, Rosenhall L, et al. Obstructive lung disease in northern Sweden: respiratory symptoms assessed in a postal survey. Eur Respir J 1991;4:257-66.

14 Baneriee DK, Lee GS, Malik SK, et al. Underdiagnosis od asthma in the elderly. Br J Dis Chest 1987;81:23-9.

\section{LUNG ALERT}

Development of mucoid Pseudomonas aeruginosa coincides with pulmonary deterioration in cystic fibrosis

$\Delta$ Li Z, Kosorok MR, Farrell PM, et al. Longitudinal development of mucoid Pseudomonas aeruginosa infection and lung disease progression in children with cystic fibrosis. JAMA 2005;293:581-8

$\mathrm{T}$

his longitudinal study correlates acquisition of non-mucoid and mucoid phenotypes of Pseudomonas aeruginosa with respiratory symptoms, quantitative chest radiography, pulmonary function tests, and anti-pseudomonal antibodies in cystic fibrosis (CF). 56 children identified by the Wisconsin CF neonatal screening project between 1985 and 1994 were followed from diagnosis (median age 6.9 weeks) for up to 16 years. Sputum or oropharyngeal swabs were cultured at least 6 monthly (mean 4 months). An anti- $P$ aeruginosa antibody titre of $\geqslant 1 / 256$ was also defined as indicative of non-mucoid $P$ aeruginosa acquisition.

Non-mucoid and mucoid $P$ aeruginosa were acquired at median ages of 1.0 and 13.0 years, respectively, with coincident increased anti-pseudomonal antibody titres. Longitudinal analysis of the time of transition from non-mucoid to mucoid status revealed a significant abrupt increase in cough score $(\mathrm{p}=0.005)$, deterioration in chest radiography (Brasfield score -2.34 of $25 ; p<0.001)$, and $12 \%(p=0.02)$ and $9 \%(p=0.007)$ reductions in predicted $\mathrm{FEV}_{1}$ and FVC, respectively. Acquisition of non-mucoid $P$ aeruginosa was not associated with significant changes in these parameters.

Anti-pseudomonal antibiotic use in 30/53 children with non-mucoid $P$ aeruginosa reduced subsequent development of mucoid phenotypes (Cox hazard ratio 0.09; $\mathrm{p}=0.001$ ). However, antibiotic treatment was also associated with increased aminoglycoside resistance in mucoid cultures $(27 \%$ v $11 \%$; $=0.02)$.

Mucoid $P$ aeruginosa expresses exopolysaccharide/alginate which confers resistance to phagocytosis and antibiotics. This study demonstrates the greater pathogenicity of mucoid $P$ aeruginosa and supports current strategies of cross infection control, early surveillance, and aggressive eradication treatment following acquisition of $P$ aeruginosa in the interval before mucoid phenotypes develop.

D Derry 